\title{
Determinación de sodio urinario como marcador bioquímico para estimar la ingesta de sal en trabajadores del Ministerio de Salud Pública y Bienestar Social, Paraguay
}

\author{
María Esther Pedrozo ${ }^{1}$ (D), Dalva De Assis ${ }^{1}$ (D) Agueda Cabello $^{1}$ (D) Felicia $^{3}$ \\ Cañete $^{2}$, Franklyn Prieto Alvarado3 iD, Noël C. Barengo ${ }^{3,4}$ iD \\ ${ }^{1}$ Ministerio de Salud Pública y Bienestar Social, Programa de Entrenamiento en Epidemiología de \\ Campo. Dirección General de Vigilancia de la Salud. Asunción, Paraguay \\ ${ }^{2}$ Ministerio de Salud Pública y Bienestar Social, Dirección de Vigilancia de Enfermedades No \\ Transmisibles. Asunción, Paraguay \\ ${ }^{3}$ Task Force for Global Health Inc. Atlanta, USA \\ ${ }^{4}$ University of Helsinki, Clinicum Department of Public Health. Helsinki, Finland
}

Cómo referenciar este artículo/ How to reference this article:
Pedrozo ME, De Assis D, Cabello A, Cañete F, Prieto Alvarado F, Barengo NC. Determinación de sodio urinario como marcador bioquímico para estimar la ingesta de sal en trabajadores del Ministerio de Salud Pública y Bienestar Social, Paraguay. Mem. Inst. Investig. Cienc. Salud. 2021; 19(3): 61-72

\begin{abstract}
RESUMEN
En América Latina, $13 \%$ de todas las muertes y 5,1\% de los años de vida ajustados por discapacidad se deben a la hipertensión. El exceso de sodio en la dieta puede incrementar aproximadamente un $30 \%$ el riesgo de hipertensión. El objetivo fue determinar la concentración de sodio en orina de 24 de horas para estimar la ingesta de sal en trabajadores del Ministerio de Salud Pública y Bienestar Social (MSPBS). Estudio transversal, en trabajadores de 25 a 64 años $(n=397)$ del MSPBS en Asunción-Paraguay en el 2014. La información sociodemográfica y económica, así como los factores de riesgo de enfermedades cardiovasculares, se evaluaron mediante un cuestionario validado y desarrollado por la OMS. Los niveles de sodio y potasio en orina de 24 horas se midieron usando un protocolo estandarizado. La mediana de la ingesta diaria de sal fue de $13,7 \mathrm{~g}$. La mediana de la excreción de sodio en orina de 24 horas fue de $239 \mathrm{mEq}$, superando el valor de excreción de sodio recomendado en un $20 \%$. Los hombres tuvieron una excreción de sodio en orina de 24 horas más alta que las mujeres tanto en el grupo de 25 a 44 años ( $251 \mathrm{mEq} / 24$ horas frente a $218 \mathrm{mEq} /$ 24 horas) como en el grupo de 45 a 64 años ( $266 \mathrm{mEq} / 24$ horas frente a $233 \mathrm{mEq} /$ 24 horas) de los participantes del estudio. En conclusión, la ingesta de sal fue notablemente superior a la recomendada por la OMS $(<5 \mathrm{~g} / \mathrm{d})$.

Palabras clave: Ingesta de sodio, hipertensión, sodio en orina, factores de riesgo, Paraguay.

\section{Determination of urine sodium excretion as a marker of estimated salt intake in employees of the Ministry of Public Health and Social Welfare, Paraguay} Entrenamiento en Epidemiología de Campo. Dirección General de Vigilancia de la Salud. Asunción, Paraguay. 
In Latin America, $13 \%$ of all deaths and $5.1 \%$ of disability-adjusted life years are due to hypertension. An excess of sodium in the diet may increase the risk of hypertension by $30 \%$. The objective was to determine the 24 -hour urine sodium concentration to estimate salt intake in employees of the Paraguayan Ministry of Public Health and Social Welfare (MSPBS). Cross-sectional study in 25-64 years-old employees $(n=397)$ of the Ministry of Public Health and Social Welfare in Asuncion-Paraguay in 2014. Socio-demographic and -economic information as well as risk factors of cardiovascular diseases were assessed using a questionnaire validated and developed by the WHO. Sodium and potassium levels in the 24-hour urine were measured using a standardized protocol. The median salt intake per day was $13.7 \mathrm{~g}$. The median of 24hour urine sodium excretion was $239 \mathrm{mEq}$, exceeding the recommended sodium excretion value by $20 \%$. Men had a higher 24 -hour urine sodium excretion than women in both the 25-44 years-old group ( $251 \mathrm{mEq} / 24$ hours vs. $218 \mathrm{mEq} / 24$ hours) and the 45-64 years-old segment of the study participants ( $266 \mathrm{mEq} / 24$ hours vs. 233 $\mathrm{mEq} / 24$ hours). In conclusion, salt intake was remarkably higher than recommended by WHO $(<5 \mathrm{~g} / \mathrm{d})$.

Keywords: Sodium intake, hypertension, urine sodium, risk factors, Paraguay.

\section{INTRODUCCION}

Se ha encontrado evidencia convincente de estudios epidemiológicos que muestran que la hipertensión es un factor de riesgo fuerte e independiente de enfermedades no transmisibles como la enfermedad coronaria, los accidentes cerebrovasculares y las enfermedades renales ${ }^{(1)}$. En América Latina, el $13 \%$ de todas las muertes y el $5,1 \%$ de los años de vida ajustados por discapacidad se deben a la hipertensión ${ }^{(2)}$. Se ha estimado que la prevalencia de hipertensión ajustada por edad en la población adulta en América Latina varía entre 26 y $42 \%{ }^{(3-6)}$. Además, hasta el $50 \%$ de los pacientes hipertensos desconocen su condición ${ }^{(7)}$. La prevalencia de hipertensión arterial está aumentando en muchos países de América Latina como Paraguay, y la situación empeorará si no hay esfuerzos para reducirla ${ }^{(8)}$.

El sodio es un nutriente esencial, es el principal ion monovalente (93\% del total de iones) de los fluidos de la sangre. El aumento de la ingesta de sodio conduce a un transporte anormal de iones sodio a nivel celular con activación del sistema nervioso simpático y del sistema renina-angiotensina-aldosterona ${ }^{(9)}$. Un exceso de sodio en la dieta puede incrementar en aproximadamente un $30 \%$ el riesgo de hipertensión ${ }^{(10)}$. La información actual de ensayos clínicos y estudios de cohortes sugieren que una reducción del sodio en la dieta disminuye la morbilidad y mortalidad por eventos cardiovasculares ${ }^{(11)}$. Además, otros estudios sugieren que una pequeña reducción del sodio en la dieta daría como resultado un beneficio considerable para la salud de la población en general.

Estudios recientes han revelado que la ingesta de sal es alta en las Américas ${ }^{(11)}$. Se ha estimado que la ingesta diaria en Argentina, Brasil y Chile supera los 9 gramos $^{(12-}$ 14). Con el fin de reducir el riesgo de resultados de salud adversos relacionados con un exceso de sodio, la Organización Mundial de la Salud (OMS) y la Organización Panamericana de la Salud (OPS) han propuesto estrategias para reducir la ingesta de sal a menos de 5 gramos por día al final de $2020^{(5)}$. Así, se ha aconsejado a los Estados miembros que establezcan valores nacionales de referencia de la ingesta dietética de sal en la población, con el fin de monitorear las tendencias y la efectividad de una posible intervención.

El método para estimar la ingesta de sal es la determinación de sodio en orina de 24 horas, que se considera el estándar de oro en comparación con otros métodos basados en muestras de orina de tiempo cronometrados y frente a los métodos basados en los recordatorios de ingesta ${ }^{(11-13)}$. En Paraguay, si bien se han realizado estudios para estimar la ingesta de sal, tanto a partir de orina espontanea en una población adulta, o en orina de 24 horas en poblaciones jóvenes. No se han publicado mediciones urinarias de sodio, utilizando el estándar de oro, para estimar la ingesta de sal en una población adulta.

Debido a la alta prevalencia de hipertensión en Paraguay, el objetivo de este estudio fue determinar la concentración de sodio en muestras de orina de 24 de horas 
para estimar la ingesta de sal en una población de trabajadores del Ministerio de Salud Pública y Bienestar Social de Paraguay, con el fin de tener información de línea de base para implementar y evaluar intervenciones para reducir el consumo de sal en esta población en particular.

\section{MATERIAL Y MÉTODOS}

Se realizó un estudio transversal utilizando las directrices del protocolo de investigación para la determinación de sodio en orina de 24 horas desarrollado por la Organización Mundial de la Salud (OMS) y la Organización Panamericana de la Salud $(\mathrm{OPS})^{(15)}$. El protocolo incluía una encuesta validada sobre factores de riesgo de sodio en orina de 24 horas. El estudio se realizó en Asunción, Paraguay, entre julio y diciembre de 2014.

La población de estudio fue el personal ejecutivo, administrativo y operativo del Ministerio de Salud Pública y Bienestar Social de ambos sexos con edades comprendidas entre 25 y 64 años. Se excluyeron aquellos que informaron dieta hiposódica y antecedentes conocidos de insuficiencia cardíaca o renal, accidente cerebrovascular, enfermedad hepática, personas que comenzaron tratamiento con diuréticos en las últimas dos semanas o con otras afecciones que dificultarían la recolección de orina durante 24 horas. El tamaño de la muestra se calculó para obtener una muestra representativa de un total de 1908 empleados del Ministerio de Salud Pública y Bienestar Social de Paraguay. El margen de error se estableció en $5 \%$ con un nivel de confianza del $95 \%$. Considerando una tasa de aceptación del $70 \%$ para participar en el estudio, el tamaño de la muestra final fue de 457.

\section{Evaluación de factores de riesgo de enfermedad cardiovascular}

La información sociodemográfica y socioeconómica, la ingesta de sal, el consumo de tabaco y alcohol, la actividad física, el consumo de frutas y verduras y la diabetes mellitus autoreportada se evaluaron mediante un cuestionario validado ${ }^{15}$. Las entrevistas fueron realizadas por profesionales de la salud capacitados y los datos se recopilaron en dispositivos electrónicos.

Los hábitos de tabaquismo autoreportados se clasificaron en dos categorías: no fumadores y fumadores diarios. El consumo de alcohol se definió como cualquier consumo de bebidas alcohólicas dentro de los 30 días anteriores a la encuesta. La actividad física baja se clasificó como menos de 10 minutos diarios de actividad física durante el tiempo libre. Para el consumo de frutas y/o verduras se categorizó en los que consumen al menos cinco porciones de frutas o verduras por día y los que no. El nivel de educación se evaluó en las siguientes categorías: (i) escuela primaria; (ii) secundaria; (iii) Universidad incompleto; (iv) pre-universitario completo; (v) universidad completa; y (vi) educación de posgrado. Las personas con diabetes mellitus autoreportados se clasificaron como diabetes mellitus.

\section{Medidas antropométricas}

En el lugar del estudio, enfermeras especialmente capacitadas midieron la altura, el peso y la presión arterial mediante un protocolo estandarizado ${ }^{(15)}$. La altura se midió con una precisión de $0,5 \mathrm{~cm}$. El peso se midió con ropa ligera. El índice de masa corporal (IMC) se calculó como el peso en kilogramos dividido por la altura en metros al cuadrado. El sobrepeso y la obesidad se definieron con los criterios de la OMS ${ }^{(16)}$. La presión arterial se midió tres veces en posición sentada después de al menos 5 minutos de descanso. Se utilizó un dispositivo OMRON Digital Automático Brazo Hem7001-E. Los valores medios de presión arterial se calcularon a partir de la segunda y tercera medición. La hipertensión se definió como presión arterial sistólica $\geq 140$ $\mathrm{mmHg}$ o presión arterial diastólica $\geq 90 \mathrm{mmHg}$.

\section{Medidas bioquímicas}

Todos los participantes recibieron instrucciones escritas y orales sobre cómo recolectar la orina de 24 horas en un recipiente con tapa de 5 litros provisto para la recolección de orina. Se recomendó a los participantes que guardaran el recipiente en un lugar fresco y oscuro. La orina recolectada se envió diariamente al laboratorio donde se midió su volumen. Se realizó la determinación de sodio y potasio, y se determinó la excreción de creatinina y proteinuria para conocer el perfil renal de cada participante. La determinación de la concentración de sodio y potasio en orina se 
realizó con el método de electrodo selectivo de iones (analizador AVL 9180-Roche $($ ). El sodio en orina se midió en $\mathrm{mEq}$ por día. La creatinina (en sangre y orina) y la proteinuria se determinaron con un equipo automatizado de química clínica CB350i. Para la determinación de creatinina en sangre y orina se utilizó una línea líquida de reactivo de creatinina cinética AA (Wiener $L a b \AA$ ) con método cinético estandarizado de Jaffe ${ }^{(18)}$. Para calcular la excreción diaria, la ecuación fue: Peso corregido de la excreción diaria = (creatinina en orina $(\mathrm{mg} / \mathrm{dl}) \times$ volumen total $(\mathrm{ml}) \times$ $0,01)$ / peso del paciente $(\mathrm{kg})$. La ecuación para el aclaramiento de creatinina fue (creatinina (o) $\times$ volumen total $(\mathrm{ml}) \times 1,73 \mathrm{~m}^{2}$ ) / (creatinina (s) $1440 \times$ área de superficie corporal). El reactivo ProtiU / LCR marca Wiener Lab ${ }^{\circledR}$, se utilizó un método colorimétrico cuantitativo con rojo de pirogalol-molibdato, con una sensibilidad de 0,5 y una linealidad de hasta 150 mg / dl para la determinación de proteinuria.

\section{Análisis estadístico}

Los datos se analizaron utilizando Epi Info $^{\mathrm{TM}}$ 7.1.4. para Windows. Se verificó la normalidad de las variables mediante las pruebas de Kolmogorov-Smirnoff. La prueba de la $X^{2}$ se utilizó para probar las diferencias en la distribución entre las variables categorizadas. Se aplicó la prueba $T$ independiente para la distribución normal de variables y ANOVA para probar las diferencias de las variables continuas entre los diferentes grupos. Los resultados se expresan como porcentajes, medias y errores estándar / desviaciones estándar. Se utilizaron modelos de regresión logística para evaluar la asociación entre la excreción urinaria de sodio $>10 \mathrm{~g} / 24$ horas (resultado) y los hábitos de estilo de vida y los factores de riesgo cardiovascular. Se utilizaron estadísticas resumidas de Hosmer-Lemeshow para evaluar la bondad de ajuste del modelo. Se presentan las odds ratio y su intervalo de confianza del $95 \%$. El nivel de significación estadística fue de 0,05.

\section{Consideraciones éticas}

Este estudio siguió las directrices de Buenas Prácticas Clínicas y la Declaración de Helsinki. Todos los datos se han recopilado utilizando, en la medida de lo posible, cuestionarios y métodos probados previamente. Además de las muestras de orina, no se utilizaron métodos invasivos. El protocolo de estudio fue aprobado por el Comité de Ética en Investigación del Laboratorio Central de Salud Pública de Paraguay. Todos los participantes dieron el consentimiento informado por escrito antes de su participación en el estudio.

\section{RESULTADOS}

En total, 397 trabajadores aceptaron participar (87\%). Sin embargo, solo 250 participantes del estudio (55\%) recolectaron las muestras de orina en condiciones óptimas. En la Tabla 1, se presenta las características basales de la población de estudio por sexo. La edad promedio fue de 42 años y el $44 \%$ vivía en Asunción; el $49 \%$ estaba casado y el $68 \%$ tiene un nivel educativo superior a la secundaria. La prevalencia de inactividad física durante el tiempo libre fue del $45 \%$ en hombres y del $65 \%$ en mujeres. Uno de cada tres participantes tenía hipertensión (38\%). El sobrepeso u obesidad fue muy prevalente en hombres (91\%) y mujeres $(77 \%)$.

Tabla 1. Características de la línea de base de los trabajadores del Ministerio de Salud Pública y Bienestar Social según sexo, Paraguay-2014

\begin{tabular}{lccc}
\hline Características & $\begin{array}{c}\text { Hombres } \\
(\mathbf{n = 1 8 9} \\
\mathbf{\%}(\mathbf{n})\end{array}$ & $\begin{array}{c}\text { Mujeres } \\
(\mathbf{n = 2 0 8} \\
\mathbf{\%}(\mathbf{n})\end{array}$ & $\begin{array}{c}\text { Total } \\
(\mathbf{n = 3 9 7}) \\
\mathbf{\%}(\mathbf{n})\end{array}$ \\
\hline $\begin{array}{l}\text { Edad (años; desviación } \\
\text { estándar) } \\
\text { Educación }\end{array}$ & $42,0 \pm 9,7$ & $42,6 \pm 10,9$ & $42,3 \pm 10,3$ \\
\multicolumn{1}{c}{ Postgrado } & $10,6(20)$ & & \\
\multicolumn{1}{c}{ Universidad } & $15,9(30)$ & $26,0(54)$ & $18,6(74)$ \\
\hline
\end{tabular}




$\begin{array}{lccc}\begin{array}{l}\text { Pre -universitario } \\ \text { completo } \\ \text { Universidad } \\ \text { (incompleto) }\end{array} & 7,9(15) & 6,7(14) & 7,3(29) \\ \begin{array}{l}\text { Escuela secundaria } \\ \text { Escuela primaria }\end{array} & 26,4(50) & 15,4(32) & 20,7(82) \\ \text { tado civil } & 33,9(64) & 18,8(39) & 25,9(103) \\ \text { Casado/a } & 5,3(10) & 6,7(14) & 6,1(24) \\ \text { Separado/a } & 51,3(97) & 46,6(97) & 48,9(194) \\ \text { Soltero/a } & 4,2(8) & 5,8(12) & 5,0(20) \\ \text { Viudo/a } & 29,1(55) & 31,3(65) & 30,2(120) \\ \text { Convivencia/a } & 14,8(28) & 2,9(6) & 1,5(6) \\ \text { Divorciado/a } & 0,5(1) & 9,6(20) & 12,9(48) \\ & & 3,9(8) & 2,3(9)\end{array}$

\section{Salario mensual medio}

$\begin{array}{llll}\text { 1-3 salarios mínimo } & 69,8(132) & 72,3(151) & 71,3(283) \\ >3 \text { salarios mínimo } & 29,6(56) & 26,9(56) & 28,2(112)\end{array}$

\section{Hábitos de estilo de vida y factores de riesgo cardiovascular}

\begin{tabular}{|c|c|c|c|}
\hline Fumar a diario & $13,2(25)$ & $7,2(15)$ & $10,1(40)$ \\
\hline Consumo de alcohol & $71,4(135)$ & $46,6(97)$ & $58,4(232)$ \\
\hline $\begin{array}{l}\text { Ingesta diaria de frutas } \\
\text { o verduras } \\
\text { (> } 5 \text { frutas / día) }\end{array}$ & $12,2(23)$ & $9,6(20)$ & $10,8(43)$ \\
\hline $\begin{array}{l}\text { Sedentario durante el } \\
\text { tiempo libre }\end{array}$ & $45,0(85)$ & $64,9(135)$ & $55,4(220)$ \\
\hline Hipertensión & $34,7(67)$ & $39,9(83)$ & $37,8(150)$ \\
\hline Diabetes mellitus & $10,6(20)$ & $14,9(31)$ & $12,9(51)$ \\
\hline $\begin{array}{l}\text { Sobrepeso u obesidad } \\
(\text { IMC }>25 \mathrm{~kg} / \mathrm{m} 2)\end{array}$ & $90,9(160)$ & $76,8(149)$ & 83,5 (309) \\
\hline Obesidad (IMC> 30) & $53,9(95)$ & $44,9(87)$ & $38,1(113)$ \\
\hline
\end{tabular}

La mediana de la excreción de sodio en orina de 24 horas fue de $239 \mathrm{mEq}$, superando el valor de excreción de sodio recomendado en un 20\% (Tabla 2). Los hombres tienden a tener una excreción de sodio en orina de 24 horas más alta que las mujeres en ambos grupos de edad. La ingesta media de sal por día fue de 13,7 gramos. Los hombres y las mujeres de los grupos mayores tienen una mayor ingesta de sal en comparación con los grupos más jóvenes. La ingesta media diaria de sal fue de $14,4 \mathrm{~g}$. en hombres de 25 a 44 años y 15,3 g. en los 45-64 años. Las cifras correspondientes para mujeres de 25 a 44 años fueron de $12,5 \mathrm{~g}$. y para los de 45 a 64 años, $13,4 \mathrm{~g}$, respectivamente. No obstante, la mediana de la ingesta diaria de sal fue casi tres veces mayor que los niveles recomendados ( $<5 \mathrm{~g} /$ día). 
Tabla 2. Parámetros de volumen de orina, excreción de sodio en orina e ingesta de sal según sexo y grupos de edad, en trabajadores del Ministerio de Salud Pública y Bienestar Social, Paraguay-2014

\begin{tabular}{|c|c|c|c|c|c|}
\hline \multirow[b]{2}{*}{ Mediciones } & \multicolumn{2}{|c|}{ Hombres } & \multicolumn{2}{|c|}{ Mujeres } & \multirow{2}{*}{$\begin{array}{c}\text { Total } \\
(n=250)\end{array}$} \\
\hline & $\begin{array}{c}25-44 \text { años } \\
(n=57)\end{array}$ & $\begin{array}{c}\text { 45-64 años } \\
(n=60)\end{array}$ & $\begin{array}{c}25-44 \text { años } \\
(n=70)\end{array}$ & $\begin{array}{c}\text { 45-64 años } \\
(n=63)\end{array}$ & \\
\hline \multicolumn{6}{|c|}{ Volumen total de orina / $24 \mathrm{~h}$} \\
\hline $\begin{array}{l}\text { Mediana }(\mathrm{ml}) \pm \\
\mathrm{DE}^{1} \\
\text { Rango } \\
\text { (gramos / } 24 \\
\text { horas) }\end{array}$ & $2162 \pm 637$ & $2317 \pm 687$ & $1935 \pm 610$ & $2028 \pm 622$ & $2080 \pm 925$ \\
\hline \multicolumn{6}{|c|}{ Excreción de sodio/orina 24 h } \\
\hline $\begin{array}{l}\text { Mediana } \\
\text { (mEq / } 24 \text { horas) } \\
\pm \text { DE }\end{array}$ & $251 \pm 59$ & $266 \pm 67$ & $218 \pm 64$ & $233 \pm 68$ & $239 \pm 94$ \\
\hline $\begin{array}{l}\text { Rango (mEq / } 24 \\
\text { horas) }\end{array}$ & $117-446$ & $90-525$ & $97-492$ & $72-576$ & $72-576$ \\
\hline \multicolumn{6}{|c|}{ Ingesta de sal / $24 \mathrm{~h}$} \\
\hline $\begin{array}{l}\text { Mediana } \\
\text { (gramos / } 24 \\
\text { horas) } \pm \text { DE } \\
\text { Rango } \\
\text { (gramos / } 24 \\
\text { horas) }\end{array}$ & $14,4 \pm 3,4$ & $\begin{array}{l}15,3 \pm 3,9 \\
5,2-30,2\end{array}$ & $12,5 \pm 3,7$ & $\begin{array}{l}13,4 \pm 3,9 \\
4.1-33.0\end{array}$ & $13,7 \pm 5,4$ \\
\hline
\end{tabular}

En la Tabla 3, se compara la mediana de la excreción de sodio en orina según actitudes y comportamientos con respecto a la ingesta de sal por sexo. En el análisis ANOVA unidireccional, no se encontró una diferencia estadísticamente significativa en la excreción de sodio en orina según el comportamiento de agregar sal a la comida al cocinar o en la mesa. La autopercepción de la cantidad de ingesta diaria de sal no se reflejó en la excreción diaria de sodio ni en hombres ni en mujeres. Sin embargo, las mujeres que informaron una alta ingesta de sal, tenían un valor medio más alto de excreción de sodio en la orina (173 mEq/día) en comparación con las mujeres con niveles normales (196 mEq/día) o poca (135 mEq/día).

Tabla 3. Actitudes y comportamientos frente al consumo de sal según sexo en trabajadores del Ministerio de Salud Pública y Bienestar Social, Paraguay-2014

\begin{tabular}{|c|c|c|c|c|}
\hline \multirow{3}{*}{$\begin{array}{l}\text { Conocimientos, actitudes y } \\
\text { comportamientos en relación } \\
\text { con la ingesta de sal }\end{array}$} & \multicolumn{4}{|c|}{ Excreción urinaria de sodio } \\
\hline & \multicolumn{2}{|c|}{ Hombres $(n=189)$} & \multicolumn{2}{|c|}{ Mujeres $(n=208)$} \\
\hline & $\begin{array}{l}\text { Mediana (mEq / } \\
\text { día) } \pm \text { DE }\end{array}$ & valor $\mathbf{p}$ & $\begin{array}{l}\text { Mediana (mEq / } \\
\text { día) } \pm \mathrm{DE}\end{array}$ & $\begin{array}{l}\text { valor } \\
\mathbf{p}\end{array}$ \\
\hline \multicolumn{5}{|l|}{$\begin{array}{l}\text { ¿Agrega sal a sus comidas } \\
\text { una vez servidas? }\end{array}$} \\
\hline Nunca / rara vez & $150 \pm 127$ & 0,418 & $130 \pm 127$ & 0,284 \\
\hline Algunas veces & $138 \pm 147$ & & $139 \pm 144$ & \\
\hline $\begin{array}{l}\text { Muy seguido/siempre } \\
\text { ¿Agrega sal al preparar sus } \\
\text { comidas? }\end{array}$ & $150 \pm 179$ & & $149 \pm 125$ & \\
\hline Nunca / rara vez & $182 \pm 146$ & 0,231 & $134 \pm 116$ & 0,411 \\
\hline Algunas veces & $128 \pm 148$ & & $93 \pm 155$ & \\
\hline Muy seguido/siempre & $144 \pm 150$ & & $142 \pm 128$ & \\
\hline $\begin{array}{l}\text { ¿Cómo calcularía la cantidad } \\
\text { de sal que consume? }\end{array}$ & & & & \\
\hline Demasiado poca/muy poca & $140 \pm 137$ & 0,374 & $135 \pm 134$ & 0,073 \\
\hline
\end{tabular}




\begin{tabular}{lll}
\hline La cantidad correcta & $145 \pm 196$ & $124 \pm 118$ \\
Demasiada/Exagerada & $145 \pm 196$ & $171 \pm 161$ \\
\hline
\end{tabular}

En la Tabla 4, se muestra la relación entre la ingesta diaria de sal $>10 \mathrm{~g}$ en trabajadores por factores sociodemográficos y socioeconómicos tanto para hombres como para mujeres. Ninguno de los factores sociodemográficos o socioeconómicos se relacionó con una mayor posibilidad de una ingesta diaria elevada de sal. Aunque el grupo de mayor edad parecía tener una menor posibilidad de ingesta diaria de sal> 10 g, la estimación puntual no alcanzó significación estadística.

Tabla 4. Factores sociodemográficos, socioeconómicos y riesgo de ingesta diaria de sal> $10 \mathrm{~g}$ según sexo en trabajadores del Ministerio de Salud Pública y Bienestar Social, Paraguay-2014.

\begin{tabular}{|c|c|c|c|c|}
\hline \multirow{2}{*}{$\begin{array}{l}\text { Factores sociodemográficos } \\
\text { y socioeconómicos }\end{array}$} & \multicolumn{2}{|c|}{ Hombres $(n=189)$} & \multicolumn{2}{|c|}{ Mujeres $(n=208)$} \\
\hline & $O_{R^{1}}$ & $95 \%$ IC $^{b}$ & $O R^{1}$ & $95 \%$ IC \\
\hline \multicolumn{5}{|l|}{ Grupos de edad } \\
\hline 25-44 años & 1,00 & ref. ${ }^{c}$ & 1,00 & ref. \\
\hline 45-64 años & 0,77 & $0,44-1,33$ & 0,92 & $0,45-1,85$ \\
\hline \multicolumn{5}{|l|}{ Nivel educacional } \\
\hline Postgrado & 1,00 & árbitro & 1,00 & árbitro \\
\hline Universidad & 0,99 & $0,42-2,35$ & 0,95 & $0,45-1,85$ \\
\hline Universidad & 2,10 & $0,65-6,83$ & 1,40 & $0,31-6,23$ \\
\hline Universidad (no completado) & 1,23 & $0,53-2,86$ & 1,57 & $0,50-4,96$ \\
\hline Escuela secundaria & 0,72 & $0,32-1,65$ & 0,98 & $0,33-2,90$ \\
\hline Escuela primaria & 1,00 & $0,30-3,33$ & 0,75 & $0,16-3,45$ \\
\hline \multicolumn{5}{|l|}{ Estado civil } \\
\hline Casado o saliendo & 1,00 & ref. & 1,00 & ref. \\
\hline Soltero & 0,82 & $0,44-1,54$ & 0,61 & $0,26-1,42$ \\
\hline Divorciado, separado o viudo & 1,23 & $0,49-3,09$ & 0,70 & $0,24-2,05$ \\
\hline \multicolumn{5}{|l|}{ Salario mensual medio } \\
\hline 1-3 salarios mínimos & 1,00 & ref. & 1,00 & ref. \\
\hline > 3 salarios mínimos & 1,13 & $0,62-2,05$ & 1,12 & $0,52-2,42$ \\
\hline
\end{tabular}

Ninguno de los hábitos de vida o factores de riesgo de enfermedad cardiovascular pudo predecir una ingesta diaria de sal> $10 \mathrm{~g}$ (Tabla 5). Los hombres y mujeres con sobrepeso u obesidad tenían una menor probabilidad de ingesta alta de sal, pero solo las mujeres obesas tenían un riesgo menor estadísticamente significativo de consumir más de $10 \mathrm{~g}$ de sal por día en comparación con las mujeres de peso normal.

Tabla 5. Hábitos de estilo de vida, factores de riesgo cardiovascular y riesgo de ingesta diaria de sal> $10 \mathrm{~g}$ según sexo en trabajadores del Ministerio de Salud Pública y Bienestar Social, Paraguay-2014

\begin{tabular}{|c|c|c|c|c|}
\hline \multirow{2}{*}{$\begin{array}{l}\text { Hábitos de estilo de vida y } \\
\text { factores de riesgo }\end{array}$} & \multicolumn{2}{|c|}{ Hombres $(n=189)$} & \multicolumn{2}{|c|}{ Mujeres $(n=208)$} \\
\hline & $\mathbf{O R}^{1}$ & $95 \%$ IC $^{b}$ & $\mathbf{O R}^{1}$ & $95 \%$ IC \\
\hline \multicolumn{5}{|l|}{ De fumar } \\
\hline No Fumar & 1,00 & Ref. $^{c}$ & 1,00 & ref. \\
\hline Fumar a diario & 1,66 & $0,47-5,84$ & 0,00 & \\
\hline \multicolumn{5}{|l|}{ Consumo de alcohol } \\
\hline No consumo & 1,00 & ref. & 1,00 & ref. \\
\hline $\begin{array}{l}\text { Consumió alcohol en los últimos } \\
30 \text { días antes de la entrevista. }\end{array}$ & 0,43 & $0,17-1,10$ & 0,75 & $0,37-1,53$ \\
\hline
\end{tabular}


Ingesta de frutas o verduras

$\begin{array}{lllll}\geq 5 \text { porciones / día } & 1,00 & \text { ref. } & 1,00 & \text { ref. } \\ \begin{array}{l}\text { < porciones / día } \\ \text { Actividad física }\end{array} & 1,14 & 0,30-4,36 & 7,48 & 0,93-5,98 \\ <10 \text { minutos / semana } & 1,00 & \text { ref. } & 1,00 & \text { ref. } \\ >10 \text { minutos / semana } & 0,86 & 0,35-2,12 & 0,91 & 0,47-2,07\end{array}$

\section{Hipertensión}

\begin{tabular}{|c|c|c|c|c|}
\hline No & 1,00 & ref. & 1,00 & ref. \\
\hline sí & 0,97 & $0,18-5,31$ & 0,87 & $0,43-1,78$ \\
\hline \multicolumn{5}{|l|}{ Diabetes } \\
\hline No & 1,00 & ref. & 1,00 & ref. \\
\hline sí & 0,00 & $\mathrm{n} / \mathrm{A}$. & 0,49 & $0,18-1,34$ \\
\hline \multicolumn{5}{|l|}{ Obesidad } \\
\hline IMC $^{c}<25 \mathrm{~kg} / \mathrm{m}^{2}$ & 1,00 & ref. & 1,00 & ref. \\
\hline $\begin{array}{l}\text { Sobrepeso u obesidad (IMC> } 25 \\
\mathrm{~kg} / \mathrm{m}^{2} \text { ) }\end{array}$ & 0,46 & $0,14-1,50$ & 0,64 & $0,27-1,48$ \\
\hline Obesidad (IMC> $30 \mathrm{~kg} / \mathrm{m}^{2}$ ) & 0,58 & $0,23-1,46$ & 0,47 & $0,22-0,99$ \\
\hline
\end{tabular}

\section{DISCUSIÓN}

Este es el primer estudio que estimó la ingesta de sal según la excreción de sodio en orina de 24 horas en una población adulta en Paraguay. Como resultados, se encontró que la media de excreción de sodio urinario fue alta en la población de estudio $(13,7 \mathrm{~g} /$ día) casi tres veces mayor que los niveles recomendados por la OMS (<5 g / día).

En otros estudios realizados en Paraguay, con orina de 24 horas en población joven, también se observaron valores elevados de sodio urinario. En el estudio de Campanogli et al., se encontró que estudiantes universitarios (46\% prehipertensión y $3 \%$ hipertensión) tenían una ingesta de sal entre 5 y $14 \mathrm{~g} /$ día $^{(19) .}$ En tanto que, en el estudio realizado por Ortiz et al., en adultos jóvenes hospitalizados encontró una natriuria promedio de $8,8 \mathrm{~g} /$ día $^{(20)}$. En otro estudio realizado por Sequera et al., en muestras de orina espontánea a primera hora de la mañana y en ayunas en una población adulta, encontró una mediana de natriuria global de $97,5 \mathrm{mmol} / \mathrm{L}$ (RIC: $59,3-139,3$ ) , que representa un valor elevado para una muestra espontánea (aprox. $20 \mathrm{mmol} / \mathrm{L})^{(21)}$.

La excreción urinaria de sodio en las poblaciones latinoamericanas es alta y similar a las observadas en nuestro estudio con una ingesta media de sal superior a $10,4 \mathrm{~g} /$ día (d) y menor del $10 \%$ con una ingesta de sodio dentro de las recomendaciones actuales ${ }^{(12-14)}$.

La ingesta excesiva de sal en la dieta en la población en general se debe muy probablemente al consumo de alimentos procesados y conservados a los que se les agrega sal, así como al uso de sal adicional agregada durante las comidas ${ }^{(18,22)}$. Un estudio colombiano descubrió que una sola porción de ciertos alimentos procesados como carne, comida rápida (como pizzas, otros), salsas o aderezos ya proporciona la cantidad diaria recomendada de sal ${ }^{(18)}$. Además, se estima que entre el $65 \%$ y el $70 \%$ de la sal que consume la población argentina tiene su origen en alimentos procesados (22). En Paraguay, un estudio realizado por Miranda et al, encontró una cantidad excesiva de sodio (razón $>1$ respecto al total de Kcal del alimento en cuestión) en 
alimentos procesados como cereales, enlatados/envasados, lácteos y derivados, así como dentro del grupo de alimentos ultraprocesados en todas las subcategorías (congelados, golosinas, bebidas, snacks y deshidratados e instantáneos) ${ }^{(23)}$. Debido a que, en nuestro estudio no encontramos ninguna correlación entre la excreción urinaria de sodio y el uso adicional de sal durante las comidas, refuerza la hipótesis de que la principal fuente de ingesta excesiva de sal son los alimentos procesados y ultraprocesados mencionados en otros estudios.

El ensayo Dietary Approaches to Stop Hypertension (DASH) demostró con éxito hace una década que una dieta baja en sodio reduce la presión arterial ${ }^{(24-25)}$. Una ligera reducción de la ingesta de sal durante 4 o más semanas provoca una disminución significativa $y$, desde el punto de vista poblacional, una importante disminución de la presión arterial tanto en individuos hipertensos como normotensos, independientemente del sexo y grupo étnico $y$, por tanto, reducir la enfermedad cardiovascular ${ }^{(26)}$. La reducción de la sal en la dieta reduce la presión arterial, la albuminuria y la proteinuria en pacientes con enfermedad renal crónica ${ }^{(27)}$. La ingesta de sal no solo es un factor de riesgo para la hipertensión, sino que también afecta el riesgo cardiovascular debido a la rigidez arterial independientemente de los cambios en la presión arterial y la aterosclerosis ${ }^{(28-29)}$. En nuestro estudio, aproximadamente uno de cada tres participantes tenía hipertensión. Es preocupante que la ingesta dietética de sal no fuera menor en ese grupo de pacientes en particular en comparación con el total.

Existe evidencia de que la restricción del contenido de sal en los alimentos procesados combinada con las intervenciones de comunicación educativa son eficaces para reducir los efectos adversos para la salud de una ingesta excesiva de sal en la dieta ${ }^{(30)}$. El etiquetado de los alimentos también tiene un impacto beneficioso en el comportamiento de los consumidores y productores en lo que respecta a la ingesta de sal, disminuyendo la futura carga por hipertensión (31). Varios países ya han implementado estrategias para reducir el consumo de sal en la población. En Argentina, grandes empresas alimentarias han firmado convenios voluntarios con el Ministerio de Salud para reducir el contenido de sodio en los alimentos procesados ${ }^{(22)}$. La iniciativa denominada Menos Sal Más Vida requería que las empresas de alimentos redujeran entre un $5 \%$ y un $15 \%$ el contenido de sodio de cuatro grupos de alimentos: 1) carnes procesadas;2) queso y productos lácteos; 3 ) sopas y aderezos; y 4) cereales, galletas, pizza y pasta (farinácea). El Ministerio estimó que la iniciativa disminuiría 0,4 gramos (1 gramo de sal) del consumo diario de sodio y si se mantiene una estrategia de reducción progresiva de sodio durante 10 años, se rebajaría 1,8 gramos de la ingesta diaria de sodio (4,5 gramos de sal) ${ }^{(32)}$.

En Paraguay, el contenido de sal (cloruro de sodio) en los productos de panadería está regulado por ley desde el 2013. Esta ley regula la cantidad máxima de sodio en los alimentos. Obligó a la industria alimentaria a reemplazar el sodio con otros ingredientes y también hizo cumplir la información visible sobre el contenido de sal de cada producto en una etiqueta. Según el informe del Instituto Nacional de Alimentación y Nutrición del 2014, cerca del $68 \%$ de las panaderías de Paraguay lograron reducir el nivel de sodio de sus productos por debajo del límite superior tolerado (máximo $600 \mathrm{mg} / 100 \mathrm{~g}$ de producto horneado) ${ }^{(33)}$.

La principal limitación de este estudio fue la baja tasa de participación de los encuestados con respecto a la recolección de orina de 24 horas a pesar de que parecían estar muy motivados al inicio del estudio y fueron alentados a participar por sus compañeros. No obstante, este problema en particular también se ha observado en otros estudios ${ }^{(31)}$. Asimismo, nos gustaría señalar que los resultados de este estudio no pueden extrapolarse a la población general ya que no seleccionamos una muestra de población representativa sino una muestra de conveniencia de los trabajadores del Ministerio de Salud. Sin embargo, creemos que los hábitos alimenticios de los participantes de nuestro estudio no son muy diferentes a la población en general, por lo que brindan información valiosa sobre los hábitos alimenticios de consumo de sal. Además, las mediciones de sodio basadas en una única recolección de orina de 24 horas no logran determinar la variabilidad de un día a otro. 
En conclusión, este estudio descriptivo proporcionó evidencia de que el consumo de sal en los trabajadores del Ministerio de Salud de Paraguay es notablemente superior al recomendado por la OMS. Es posible que se necesiten intervenciones de atención de la salud para reducir la ingesta dietética de sal en esa población. Es necesario estudiar las concentraciones de sal de los alimentos que se ofrecen en los supermercados para definir mejor la fuente de ingesta de sal en la dieta. Además, es necesario estudiar con más detalle la asociación entre los grupos sociodemográficos y socioeconómicos y la ingesta dietética de sal no solo en los trabajadores del Ministerio de Salud, sino también en la población paraguaya. Finalmente, los cuestionarios de la encuesta de la OPS / OMS que evalúan la ingesta de sal pueden no cubrir las fuentes más importantes de sal en la dieta, por lo que sus preguntas están poco relacionadas con la excreción de sodio en la orina.

Por último, se destaca que, este estudio se utilizó en el Plan Nacional de Acción para el Control de las Enfermedades Crónicas No Trasmisibles 2014-2024, como línea de base para el consumo medio de sal en la población (estimado 13 gramos/día), cuya meta para el 2024 es la Reducción relativa del 30\% del consumo medio de sal diaria en la población adulta (Meta: consumo medio de sal 9,1 gramos / día) ${ }^{(35)}$.

\section{AGRADECIMIENTOS}

Nos gustaría agradecer a las siguientes personas por su contribución en la implementación de este estudio: Dra. Margarita Dacack (Laboratorio del Hospital Distrital de Ñemby), Dra. Malvina Páez, Lic. Fátima Vázquez, Lic. Dora Ramírez, Lic. Sandra Ocampos, Lic. Silvana Rotela, Dra. Rosa Galeano, Lic. Ethel Santacruz y Bioq. Jorge Ovando.

Aprobación ética: El protocolo del estudio fue aprobado por el Comité de Ética en Investigación del Laboratorio Central de Salud Pública de Paraguay.

Financiamiento: Este proyecto fue financiado en insumos y reactivos por una beca de la TEPHINET/Centers for Disease Control and Prevention (CDC)/ Task Force for Global Health, Inc. ("TFGH"), Georgia, United States of America. La contracomparte fue desarrollada con recursos y personal de la DGVS-MSPBS.

Conflictos de intereses: Ninguno declarado.

Contribución de autores: María Esther Pedrozo, Dalva De Assis, Agueda Cabello, Felicia Cañete y Franklyn Prieto, Alvarado participaron en la concepción y diseño del estudio e interpretación de los datos, revisando el artículo y aprobando la versión final a presentar. Noël C. Barengo contribuyó con el análisis e interpretación de los datos, revisó críticamente el artículo y aprobó la versión final que se presentará.

Aprobación ética: El protocolo del estudio fue aprobado por el Comité de Ética en Investigación del Laboratorio Central de Salud Pública de Paraguay.

\section{REFERENCIAS BIBLIOGRÁFICAS}

1. Collins $R$, Peto $R$, MacMahon $S$, Hebert $\mathrm{P}$, Fiebach $\mathrm{NH}$, Eberlein $\mathrm{KA}$ et al. Blood pressure, stroke and coronary heart disease. Part 2: short term reductions in blood pressure: overview of randomised drug trials in their epidemiological context. Lancet 1990; 335: 827-39.

2. Lawes CM, Vander Hoorn S, Rodgers A; International Society of Hypertension Global burden of blood-pressure-related disease, 2001. Lancet 2008; 371(9623): 1513-8.
3. Burlando G, Sánchez RA, Ramos $F H$, Mogensen CE, Zanchetti A, Latin American Experts Group. Latin American consensus on diabetes mellitus and hypertension. J Hypertens 2004; 22(12): 2229-41.

4. Albala C, Vio F, Kain J, Uauy R. Nutrition transition in Latin America: the case of Chile. Nutr Rev 2001; 59(6): 170-6.

5. Lanas $F$, Avezum A, Bautista LE, Díaz R, Luna $M$, Islam $S$, et al. Risk Factors for Acute Myocardial Infarction in Latin America The INTERHEART Latin 
American Study. Circulation 2007; 115(9):1067-74.

6. de Sereday MS, Gonzalez C, Giorgini D, De Loredo L, Braguinsky J, Cobeñas C, et al. Prevalence of diabetes, obesity, hypertension and hyperlipidemia in the central area of Argentina. Diabetes Metab 2004; 30(4):335-9.

7. Hart JT. Rule of halves: implications of increasing diagnosis and reducing dropout for future workload and prescribing costs in primary care. $\mathrm{Br}$ ] Gen Pract J R Coll Gen Pract 1992; 42(356): 116-9.

8. Rubinstein A, Alcocer L, Chagas A. High blood pressure in Latin America: a call to action. Ther Adv Cardiovasc Dis. 2009; 3(4):259-85.

9. Liu $Y$, Li $H$, Hong $S$, Yin $X$. Salt reduction and hypertension in China: a concise state-of-the-art review. Cardiovasc Diagn Ther 2015; 5(3):1916.

10. Intersalt: an international study of electrolyte excretion and blood pressure. Results for 24 hour urinary sodium and potassium excretion. Intersalt Cooperative Research Group. BMJ 1988; 297(6644):319-28.

11. Whelton PK. Sodium, potassium, blood pressure, and cardiovascular disease in humans. Curr Hypertens Rep. 2014; 16(8):465.

12. Rodrigues $S L$, Souza Júnior $P R$, Pimentel EB, Baldo MP, Malta DC, Mill $J G$, et al. Relationship between salt consumption measured by 24-h urine collection and blood pressure in the adult population of Vitória (Brazil). Braz J Med Biol Res 2015; 48(8):728-35.

13. López-Rodríguez G, Galván-García $M$, Muzzo B S. Urinary sodium excretion in children and adults from a borough of the metropolitan region of Chile. Rev Chil Nutr 2009; 36(4):1139-43.

14. Cornejo Karen P F, Atalah E., Galgani, J. Assessment of dietary intake and urinary excretion of sodium and potassium in adults. Revista médica de Chile 2014; 142: 687-95.

15. Panamerican Health Organization. Salt Smart Americas. Guide for action in the countries. Techincal document Washington, DC; 2013. Available at: http://www.audyn.org.uy/sitio/repo/arc h/ReduccionsalAmericasdigital 1.pdf

16. Whitworth JA; World Health Organization, International Society of Hypertension Writing Group. 2003 World Health Organization (WHO)/International Society of Hypertension (ISH) statement on management of hypertension. J Hypertens. 2003; 21(11):1983-92.
17. Bingham S. The dietary assessment of individuals; methods, accuracy, new techniques and recommendations. Nutr Abstr Rev A 1987; 57: 706-42.

18. Garcés C, Cristina I, Ramírez G, Davahiva B, Charry G, Alejandro D. Sodium content in processed foods from Colombia evaluated by nutritional labeling. Nutr Humana 2014; 16(1):6182.

19. Ferrante D, Apro N, Ferreira V, Virgolini $M$, Aguilar V. Feasibility of salt reduction in processed foods in Argentina. Rev Panam Salud Publica 2011;29: 69-75

20. Campagnoli T., González L., Santa Cruz F. Salt intake and blood pressure in the University of Asuncion-Paraguay youths: a preliminary study. Braz. J. Nephrol. 34 (4), [Internet] Dec 2012. [Citado octubre 2015]. Disponible en: https://doi.org/10.5935/01012800.20120026

21. Ortiz J., Aveiro A., Ortega E. Relación entre la excreción urinaria de sodio, la presión arterial y el índice de masa corporal en adultos-jóvenes. Rev. Nac. (Itauguá) [Internet]. 2019 Jun [citado 2020 Nov 21]; 11(1): 39-55. Disponible en: http://scielo.iics.una.py/scielo.php?scrip $\mathrm{t}=\mathrm{sci}$ arttext\&pid $=\mathrm{S} 2072$ -

$81742019000100039 \& \operatorname{lng}=e s$. https://doi.org/10.18004/rdn2019.0011 .01.039-055.

22. Sequera VG, Cañete $F$, Paiva $T$, Giménez E, Santacruz E, Fretes G et al . Patrones de excreción urinaria de Sodio en población adulta en muestras de orina espontánea. An. Fac. Cienc. Méd. (Asunción) [Internet]. 2017 Abr [citado 2021 Set 11]; 50(1): 51-60. Disponible en:

http://scielo.iics.una.py/scielo.php?scrip $\mathrm{t}=\mathrm{sci}$ arttext\&pid $=\mathrm{S} 1816$ $89492017000100051 \& \operatorname{lng}=e s$. https://doi.org/10.18004/anales/2017.0 50(01)51-060.

23. Miranda Eliana Meza, Nuñez Beatriz Elizabeth, Maldonado Olga. Evaluación de la composición nutricional de alimentos procesados y ultraprocesados de acuerdo al perfil de alimentos de la Organización Panamericana de la Salud, con énfasis en nutrientes críticos. Mem. Inst. Investig. Cienc. Salud [Internet]. 2018 Abr [citado 2021 Oct 19]; 16(1): 54-63. Disponible en: https://doi.org/10.18004/mem.iics/181 2-9528/2018.016(01)54-063.

24. Conlin PR, Chow D, Miller ER 3rd, Svetkey LP, Lin PH, Harsha DW, et al. The effect of dietary patterns on blood pressure control in hypertensive patients: results from the Dietary 
Approaches to Stop Hypertension (DASH) trial. Am J Hypertens. 2000; 13(9):949-55.

25. Sacks FM, Svetkey LP, Vollmer WM, Appel LJ, Bray GA, Harsha D, et al; DASH-Sodium Collaborative Research Group. Effects on blood pressure of reduced dietary sodium and the Dietary Approaches to Stop Hypertension (DASH) diet. DASH-Sodium Collaborative Research Group. N Engl J Med. 2001; 344(1):3-10.

26. He FJ, Li J, Macgregor GA. Effect of longer-term modest salt reduction on blood pressure. Cochrane Database Syst Rev 2013; 4: CD004937.

27. McMahon EJ, Bauer JD, Hawley CM, Isbel NM, Stowasser M, Johnson DW, et al. A Randomized Trial of Dietary Sodium Restriction in CKD. J Am Soc Nephrol 2013; 24(12):2096-103.

28. Azak A, Huddam B, Gonen N, Yilmaz SR, Kocak G, Duranay M. Salt Intake Is Associated with Inflammation in Chronic Heart Failure. Int Cardiovasc Res J 2014; 8(3):89-93.

29. Cook NR, Appel LJ, Whelton PK. Lower Levels of Sodium Intake and Reduced Cardiovascular Risk. Circulation 2014; 129(9):981-9.

30. Smith-Spangler CM, Juusola JL, Enns EA, Owens DK, Garber AM. Population strategies to decrease sodium intake and the burden of cardiovascular disease: a cost-effectiveness analysis. Ann Intern Med 2010; 152(8): 481-7.
31. Nghiem N, Blakely T, Cobiac LJ, Pearson $A L$, Wilson N. Health and economic impacts of eight different dietary salt reduction interventions. PLoS One 2015; 10(4):e0123915.

32. Konfino J, Mekonnen TA, Coxson PG, Ferrante D, Bibbins-Domingo $\mathrm{K}$. Projected impact of a sodium consumption reduction initiative in Argentina: an analysis from the CVD policy model-Argentina. PLoS One. 2013; 8(9):e73824.

33. Instituto Nacional de Alimentación y Nutrición. Niveles de Sodio por cantidad de muestras panificados recolectadas de 7 Departamentos del Paraguay. 2014. Available at http://inan.gov.py/newweb/?page_id=5 34

34. Ji C, Sykes L, Paul C, Dary O, Legetic B, Campbell NRC, et al. Systematic review of studies comparing 24-hour and spot urine collections for estimating population salt intake. Rev Panam Salud Pública 2012; 32(4):307-15.

35. Paraguay. Ministerio de Salud Pública y Bienestar Social. Dirección General Vigilancia de la Salud. Dirección de Vigilancia de Enfermedades No Transmisibles. Plan Nacional de Acción para la Prevención y el Control de las Enfermedades Crónicas No Transmisibles. 2014- 2024. Asunción: MSP y BS, 2014. 36 pág. Disponible en: http://168.90.176.14:8011/wpcontent/uploads/2021/05/Plan-Accionde-ECNT-2014.pdf 